\title{
An Optimal Dimensionality Sampling Scheme on the Sphere with Accurate and Efficient Spherical Harmonic Transform for Diffusion MRI
}

\author{
Alice P. Bates, Student Member, IEEE, Zubair Khalid, Member, IEEE and Rodney A. Kennedy, Fellow, IEEE
}

\begin{abstract}
We design a sampling scheme on the sphere and a corresponding spherical harmonic transform (SHT) for the measurement and reconstruction of the diffusion signal in diffusion magnetic resonance imaging (dMRI). By exploiting the antipodal symmetry property of the diffusion signal in the spectral (spherical harmonic) domain, we design a sampling scheme that attains the optimal number of samples, equal to the degrees of freedom required to represent the antipodally symmetric band-limited diffusion signal in the spectral domain. Compared with other sampling schemes that can be used with the optimal number of samples, we demonstrate, through numerical experiments, that the proposed scheme enables more accurate computation of the SHT, and this accuracy is practically rotationally invariant. In addition, it results in more efficient computation of the SHT and storage of the diffusion signal.
\end{abstract}

Index Terms-diffusion magnetic resonance imaging; sampling; spherical harmonic transform; antipodal signal; sphere.

\section{INTRODUCTION}

In diffusion magnetic resonance imaging (dMRI), the diffusion signal measurements in each voxel are collected on a spherical sampling grid or multiple concentric spherical sampling grids in $q$-space [1]-[7], where $q$ is the diffusion wave vector. The reconstruction of the diffusion signal from these measurements is achieved by expanding the signal in the spherical harmonic basis-a complete orthonormal basis on the sphere [8]. The expansion in spherical harmonic basis is enabled by the spherical harmonic transform (SHT).

For the accurate reconstruction and spectral analysis in the spherical harmonic domain of the diffusion signal, it is necessary to design the sampling scheme such that the SHT can be computed accurately from the measurements. As fibres may assume any orientation, a sampling scheme design should allow for a reconstruction accuracy that is independent of the orientation of the diffusion signal or the sampling grid (rotationally invariant) [1], [9]. Since the number of measurements of the diffusion signal that can be acquired is heavily restricted by the scan time, it is also important that fewer samples are required by a sampling scheme [10], [11]. In order for a sampling scheme to allow for the accurate computation of the SHT, the minimum number of samples required is

A. P. Bates and R. A. Kennedy are with the Research School of Engineering, College of Engineering and Computer Science, The Australian National University, Canberra, Australia. Z. Khalid is with the Department of Electrical Engineering, School of Science and Engineering, Lahore University of Management Sciences, Lahore 54792, Pakistan. This work is supported by the Australian Research Council's Discovery Projects funding scheme (Project no. DP150101011). E-mail: alice.bates@ anu.edu.au; zubair.khalid@lums.edu.pk; rodney.kennedy@anu.edu.au equal to the degrees of freedom of the diffusion signal in the spectral domain; this number is referred as the optimal spatial dimensionality or optimal dimensionality for short [12]. For the diffusion signal band-limited at $L$ (defined in Section II), the optimal dimensionality is $N_{\mathrm{O}}=L(L+1) / 2$ [13]. Furthermore, computational complexity and storage requirements of the measurement and reconstruction process are important considerations in the design of a sampling scheme [14].

Electrostatic energy minimisation [1], [5], tessellation of the sphere [15], [16] and spherical code [2] are some of the methods used to achieve a sampling grid with uniform sampling and antipodal symmetry. These methods, can be used to construct sampling grids of arbitrary size (with the exception of spherical tessellation), however they only allow for approximate computation of the SHT using least-squares, where the approximation improves with a larger number of samples [17]. The least-squares method is also computational intensive compared with fast SHT algorithms [12], [18]. The spherical design with uniform density sampling method [10] has a uniform and antipodally symmetric arrangement of samples, and allows for the accurate computation of the SHT, however it requires more than $N_{\mathrm{O}}$ samples [17].

An equiangular scheme [18] with $2 L^{2}$ samples that allows accurate and efficient computation of the SHT has been used in dMRI [3]. An iso-latitude scheme with $L^{2}$ samples, the optimal dimensionality of an arbitrary (without antipodal symmetry) band-limited signal on the sphere, has also been developed [12], which allows the accurate and fast computation of the SHT. However, the samples in both of these schemes are neither antipodally symmetric nor uniform by design. In this work, we focus on the recently proposed sampling scheme [13] which exploits the antipodal symmetry property of the diffusion signal in the spatial domain to place the samples on the sphere such that the SHT in [12] can be used with the optimal number of samples. We refer to this scheme as the spatial antipodal sampling scheme.

In this work, we address the following research questions:

- Can we exploit the antipodal symmetry property of the diffusion signal in the spectral domain to design a sampling scheme with optimal spatial dimensionality and which allows for an accurate SHT?

- Does the proposed scheme allow for superior reconstruction of the diffusion signal compared with the spatial antipodal sampling scheme, the only scheme that has $N_{\mathrm{O}}$ samples and allows for the accurate computation of the SHT, in terms of accuracy of reconstruction, computation 
complexity of the SHT and storage requirements? Does the scheme achieve rotationally invariant reconstruction?

In addressing these questions, we organise the rest of the paper as follows. We review the necessary mathematical background for the diffusion signal and spherical harmonics in Section II, where we also review the spatial antipodal sampling scheme. The proposed sampling scheme and SHT is presented in Section III. In Section IV, we carry out the analysis of reconstruction accuracy, rotational invariance, computational complexity and storage requirements of the SHT associated with the proposed sampling scheme. Concluding remarks are then made in Section V.

\section{Preliminaries}

\section{A. Diffusion Signal on the Sphere}

The diffusion signal at a fixed $\boldsymbol{q}$-space radius represents a signal on the sphere. Let the diffusion signal be denoted by $d(\theta, \phi)$, where $\theta \in[0, \pi]$ is the co-latitude and $\phi \in$ $[0,2 \pi)$ denotes the longitude, which parameterise a point $(\sin \theta \cos \phi, \sin \theta \sin \phi, \cos \theta)^{\prime} \in \mathbb{R}^{3}$ on the unit sphere $\mathbb{S}^{2}$.

Remark 1 (On the antipodal symmetry property of the diffusion signal in the spatial domain): The diffusion signal has the property that it is antipodally symmetric; in the spatial domain the diffusion signal has the same value at locations diametrically opposite each other with $d(\theta, \phi)=d(\pi-\theta, \phi+\pi)$.

\section{B. Spherical Harmonic Expansion}

The spherical harmonic functions (or spherical harmonics for short) form a complete basis for $L^{2}\left(\mathbb{S}^{2}\right)$ and are defined as [8]

$$
Y_{\ell}^{m}(\theta, \phi)=\sqrt{\frac{2 \ell+1}{4 \pi} \frac{(\ell-m) !}{(\ell+m) !}} P_{\ell}^{m}(\cos \theta) e^{i m \phi},
$$

for integer degree $\ell \geq 0$ and integer order $|m| \leq \ell$. In (1), $P_{\ell}^{m}$ denotes the associated Legendre function of degree $\ell$ and order $m$ [8].

Due to completeness of spherical harmonics, we can expand the diffusion signal as [8]

$$
d(\theta, \phi)=\sum_{\ell=0}^{\infty} \sum_{m=-\ell}^{\ell}(d)_{\ell}^{m} Y_{\ell}^{m}(\theta, \phi),
$$

where $(d)_{\ell}^{m}$ denotes the spherical harmonic coefficient of degree $\ell$ and order $m$ and is given by the spherical harmonic transform (SHT) defined as

$$
(d)_{\ell}^{m} \triangleq \int_{\mathbb{S}^{2}} d(\theta, \phi) \overline{Y_{\ell}^{m}(\theta, \phi)} \sin \theta d \theta d \phi .
$$

The spherical harmonic coefficients $(d)_{\ell}^{m}$ constitute the spectral domain representation of the diffusion signal.

Remark 2 (On the antipodal symmetry property of the diffusion signal in the spectral domain): Since $Y_{\ell}^{m}(\theta, \phi)=$ $Y_{\ell}^{m}(\pi-\theta, \pi+\phi)$ for even $\ell$ and $Y_{\ell}^{m}(\theta, \phi)=-Y_{\ell}^{m}(\pi-$ $\theta, \pi+\phi)$ for odd $\ell$, (Remark 1) implies that $(d)_{\ell}^{m}=0$ for odd degree $\ell$ [10], [13], [19].

In this work, we assume that the diffusion signal is bandlimited $^{1}$ at degree $L$ such that $(d)_{\ell}^{m}=0$ for $\ell \geq L$. With

\footnotetext{
${ }^{1}$ The band-limit $L$ required to accurately represent the diffusion signal depends on the $\boldsymbol{q}$-space radius [3], [4], [19].
}

this consideration and following Remark 2, we rewrite the expansion of the diffusion signal in (2) as

$$
d(\theta, \phi)=\sum_{\substack{\ell=0 \\ \ell \text { even }}}^{L-1} \sum_{m=-\ell}^{\ell}(d)_{\ell}^{m} Y_{\ell}^{m}(\theta, \phi), \quad L \text { odd } .
$$

We refer to the reconstruction of the diffusion signal from its spherical harmonic coefficients, given in (4), as the inverse SHT.

Remark 3 (Optimal dimensionality of the sampling scheme for diffusion signal): The number of spherical harmonic coefficients required to represent the diffusion signal, given in (4), is $N_{\mathrm{O}}=L(L+1) / 2$ [19], [20], which also represents the optimal dimensionality, defined as the number of samples attainable by any sampling scheme that allows the accurate computation of the SHT of any band-limited antipodal signal on the sphere.

\section{Spatial Antipodal Sampling Scheme}

We briefly review the spatial antipodal sampling scheme [13] which customises the sampling scheme [12] for acquisition of measurements of the diffusion signal in dMRI. We first revisit the optimal dimensionality sampling scheme [12], followed by the review of the spatial antipodal sampling scheme [13].

The optimal dimensionality sampling scheme has an isolatitude sampling grid [12]; the $L$ locations along $\theta$ where the iso-latitude rings are placed are stored in the vector $\boldsymbol{\theta} \triangleq\left[\theta_{0}, \theta_{1}, \ldots, \theta_{L-1}\right]^{T}$. The scheme has $2 n+1$ equally spaced samples along longitude in the ring placed at $\theta_{n} \in \boldsymbol{\theta}$ with $\phi^{n} \triangleq\left[0, \Delta_{n}, 2 \Delta_{n}, \ldots,(2 n) \Delta_{n}\right], \quad \Delta_{n}=\frac{2 \pi}{2 n+1}$. The sampling grid, formed by $\boldsymbol{\theta}$ and $\phi^{n}$, is composed of $L^{2}$ samples, which is equal to the number of degrees of freedom required to represent any signal band-limited at degree $L$.

The spatial antipodal sampling scheme [13] uses an antipodal placement of samples on the sphere; the iso-latitude rings in $\boldsymbol{\theta}$ are placed in pairs antipodal to one another, with the samples in the ring $\theta_{n}$ antipodal to the samples in the ring $\theta_{n-1}$ for $n=2,4, \ldots, L-1$, that is

$$
\boldsymbol{\theta} \triangleq\left[0, \ldots, \pi-\theta_{L-3}, \theta_{L-3}, \pi-\theta_{L-1}, \theta_{L-1}\right]^{T}, \quad L \text { odd } .
$$

This arrangement means that measurements only need to be taken over the rings $\theta_{n}, n=0,2, \ldots, L-1$. Samples are equally spaced along longitude, with $k$-th sample location, denoted by $\phi_{k}^{n}$, in the ring placed at $\theta_{n}$ given by

$$
\phi_{k}^{n} \triangleq\left\{\begin{array}{lll}
\frac{2 k \pi}{2 n+1}, & n=0,2, \ldots, L-1, & k \in[0,2 n], \\
\frac{\pi(2 k+1)}{2 n+3}, & n=1,3, \ldots, L-2, & k \in[0,2(n+1)] .
\end{array}\right.
$$

Since the measurements are only required to be taken over $(L+1) / 2$ rings due to antipodal symmetry (Remark 1$)$, the antipodal scheme requires $L(L+1) / 2=N_{\mathrm{O}}$, in total, for the computation of the SHT. Thus the spatial antipodal sampling scheme achieves the optimal dimensionality. 


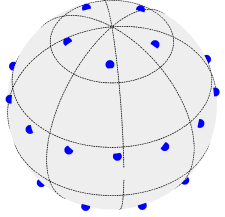

(a)

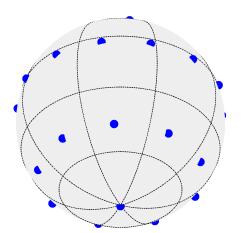

(b)
Fig. 1: (a) North pole view and (b) South pole view of the proposed sampling scheme on the sphere given by (5) and (6) for measuring diffusion signal $d(\theta, \phi)$ band-limited at $L=7$.

\section{Proposed Sampling Scheme and Spherical HARMONIC TRANSFORM}

In order to answer Q1, posed in Section I, we use the antipodal symmetry property of the diffusion signal in the spectral domain to customise the optimal dimensionality sampling scheme [12] to design a sampling scheme that achieves optimal dimensionality and allows accurate computation of the SHT. As with [13], we propose that measurements are taken over $(L+1) / 2$ iso-latitude rings. However, rather than using the antipodal symmetry property of the diffusion signal in the spatial domain (Remark 1) to determine the value of the diffusion signal on the remaining $(L-1) / 2$ rings, we redesign the SHT algorithm proposed in [12]. By exploiting the antipodal symmetry property in the spectral domain (Remark 2 ), the proposed SHT only requires diffusion signal values on $(L+1) / 2$, rather than $L$, iso-latitude rings. We refer to the proposed scheme as the spectral antipodal sampling scheme.

\section{A. Proposed Sampling Grid}

We propose an iso-latitude sampling scheme, denoted by $\mathfrak{S}(L)$, with $(L+1) / 2$ iso-latitude rings, located at

$$
\boldsymbol{\theta} \triangleq\left[\theta_{0}, \theta_{1}, \ldots, \theta_{(L-1) / 2}\right]^{T}, \quad L \text { odd }
$$

and sample equally spaced along longitude, with $k$-th sample location, denoted by $\phi_{k}^{n}$, in the ring placed at $\theta_{n}$ given by

$$
\phi_{k}^{n} \triangleq \frac{2 k \pi}{4 n+1}, \quad n \in[0,(L-1) / 2], \quad k \in[0,4 n] .
$$

As an example, Fig. 1 shows the proposed scheme for $L=7$.

Remark 4 (On the dimensionality of proposed sampling scheme): Since the total number of samples in the proposed scheme $\mathfrak{S}(L)$ are

$$
\sum_{n=0}^{(L-1) / 2}(4 n+1)=\frac{(L+1) L}{2}=N_{\mathrm{O}} .
$$

the proposed scheme attains the optimal spatial dimensionality.

\section{B. Proposed Spherical Harmonic Transform (SHT)}

We propose a SHT over the proposed sampling grid, defined by the vectors $\boldsymbol{\theta}$ and $\phi_{k}^{n}$, given in (7) and (8). We define a vector $\boldsymbol{\theta}^{m} \triangleq\left[\theta_{|m / 2|}, \theta_{|m / 2|+1}, \ldots, \theta_{(L-1) / 2}\right]^{T} \subset$ $\boldsymbol{\theta} \quad|m|<L, m$ even and $\boldsymbol{\theta}^{m} \triangleq \boldsymbol{\theta}^{m+1} \quad m$ odd. The vector $\mathbf{g}_{m} \equiv G_{m}\left(\boldsymbol{\theta}^{m}\right)$, with

$$
G_{m}\left(\theta_{n}\right) \triangleq \int_{0}^{2 \pi} f\left(\theta_{n}, \phi\right) e^{-i m \phi} d \phi=2 \pi \sum_{\substack{\ell=|m| \\ \ell \text { even }}}^{L-1}(f)_{\ell}^{m} \widetilde{P}_{\ell}^{m}\left(\theta_{n}\right),
$$

is defined for $|m|<L$ and $\theta_{n} \in \boldsymbol{\theta}$, where $\widetilde{P}_{\ell}^{m}\left(\theta_{n}\right) \triangleq$ $Y_{\ell}^{m}\left(\theta_{n}, 0\right)$. The integral in (10) can be accurately evaluated as a summation provided there are at least $2 m+1$ samples along $\phi$ [12]. As samples are equally spaced around $\phi(8), G_{m}\left(\theta_{n}\right)$ for $\theta_{n} \in \boldsymbol{\theta}^{m}$ can be computed using the FFT, then the spherical harmonic coefficients of order $m$ can be recovered from (10) by setting up a system of linear equations, given by

$$
\mathbf{g}_{m}=\mathbf{P}_{L}^{m} \mathbf{f}_{m}, \quad|m| \leq L,
$$

where

$$
\mathbf{f}_{m}=\left\{\begin{array}{l}
{\left[(f)_{|m|}^{m},(f)_{|m|+2}^{m}, \ldots,(f)_{L-1}^{m}\right]^{T}, \quad m \text { even, }} \\
{\left[(f)_{|m|+1}^{m},(f)_{|m|+3}^{m}, \ldots,(f)_{L-1}^{m}\right]^{T}, \quad m \text { odd },}
\end{array}\right.
$$

and $\mathbf{P}_{L}^{m}$ is defined as

$$
\mathbf{P}_{L}^{m} \triangleq 2 \pi\left(\begin{array}{cccc}
\widetilde{P}_{|m|}^{m}\left(\theta_{s}\right) & \widetilde{P}_{|m|+2}^{m}\left(\theta_{s}\right) & \cdots & \widetilde{P}_{L-1}^{m}\left(\theta_{s}\right) \\
\widetilde{P}_{|m|}^{m}\left(\theta_{s+1}\right) & \widetilde{P}_{|m|+2}^{m}\left(\theta_{s+1}\right) & \cdots & \widetilde{P}_{L-1}^{m}\left(\theta_{s+1}\right) \\
\vdots & \vdots & \ddots & \vdots \\
\widetilde{P}_{|m|}^{m}\left(\theta_{\frac{L-1}{2}}\right) & \widetilde{P}_{|m|+2}^{m}\left(\theta_{\frac{L-1}{2}}\right) & \cdots & \widetilde{P}_{L-1}^{m}\left(\theta_{\frac{L-1}{2}}\right)
\end{array}\right),
$$

for even $m$ and

$$
\mathbf{P}_{L}^{m} \triangleq 2 \pi\left(\begin{array}{cccc}
\widetilde{P}_{|m|+1}^{m}\left(\theta_{s}\right) & \widetilde{P}_{|m|+3}^{m}\left(\theta_{s}\right) & \cdots & \widetilde{P}_{L-1}^{m}\left(\theta_{s}\right) \\
\widetilde{P}_{|m|+1}^{m}\left(\theta_{s+1}\right) & \widetilde{P}_{|m|+3}^{m}\left(\theta_{s+1}\right) & \cdots & \widetilde{P}_{L-1}^{m}\left(\theta_{s+1}\right) \\
\vdots & \vdots & \ddots & \vdots \\
\widetilde{P}_{|m|+1}^{m}\left(\theta_{\frac{L-1}{2}}\right) & \widetilde{P}_{|m|+3}^{m}\left(\theta_{\frac{L-1}{2}}\right) & \cdots & \widetilde{P}_{L-1}^{m}\left(\theta_{\frac{L-1}{2}}\right)
\end{array}\right),
$$

for odd $m$. Here $s=\lceil|m| / 2\rceil$, where $\lceil\cdot\rceil$ denotes the integer ceiling function. We note that the size of system of linear equations given in (11) is $\lceil(L-|m|) / 2\rceil$, rather than $L-|m|$ for the case of spatial antipodal sampling scheme [13].

\section{Placement of Iso-latitude Rings}

In order to accurately compute the SHT, we require the sampling points along co-latitude to be chosen such that the matrix $\mathbf{P}_{L}^{m}$ is well-conditioned for each $|m| \leq L$ [12], [13]. We propose the following method to achieve this: define a set of equiangular $(L+1) / 2$ samples along co-latitude given by $\Theta=\left\{\frac{\pi(2 t+1)}{L}\right\}, \quad t=0,1, \ldots, \frac{L-1}{2}$, then the optimal ordering is selected by first choosing the location of the ring of $2 L-1$ (the largest number of) samples along $\phi$ from the set $\Theta$ as farthest from the poles, then for each $m=L-$ $3, L-5, \ldots 2$, choose $\theta_{m / 2}$ from the set $\Theta$ that minimises the sum of the condition numbers of the matrices $\mathbf{P}_{L}^{m}$ and $\mathbf{P}_{L}^{m-1}$ and finally set $\theta_{0}=\pi$. Such placement of samples along co-latitude results in $\mathbf{P}_{L}^{m}$ being well-conditioned, which ensures the accurate computation of the SHT. We analyse the accuracy of the SHT in the next section.

\section{Evaluation of Spectral Antipodal Optimal DimENSIONALITY SAMPLING SCHEME}

In order to address Q2 posed in Section I, we now investigate whether the proposed scheme performs better in terms of reconstruction accuracy, and computational and storage efficiency than the spatial antipodal sampling scheme. 


\section{A. Numerical Accuracy}

The following experiment is conducted to evaluate the numerical accuracy of the SHT associated with the proposed sampling scheme and the effect of rotation of the signal on the numerical accuracy.

A band-limited antipodally symmetric test signal $f_{\mathrm{t}}$ is synthesised in the spectral domain by generating spherical harmonic coefficients $\left(f_{\mathrm{t}}\right)_{\ell}^{m}$ for $0<\ell<L$, $\ell$ even, $|m| \leq \ell$ with real and imaginary parts uniformly distributed in the interval $[-1,1]$. The test signal $f_{\mathrm{t}}$ is then obtained in the spatial domain over the proposed sampling grid $\mathfrak{S}(L)$ using the inverse SHT, followed by the SHT to get the spherical harmonic coefficients of the reconstructed signal, $\left(f_{\mathrm{r}}\right)_{\ell}^{m}$. For each band-limit $1 \leq L \leq 25$ (band-limits of interest in dMRI), we repeat the experiment 10 times and record the average value of the mean reconstruction error $E_{\text {mean }}$, given by

$$
E_{\text {mean }} \triangleq \frac{1}{L^{2}} \sum_{\ell=0}^{L-1} \sum_{m=-\ell}^{\ell}\left|\left(f_{\mathrm{t}}\right)_{\ell}^{m}-\left(f_{\mathrm{r}}\right)_{\ell}^{m}\right| .
$$

In order to analyse the effect of rotation on the accuracy of the SHT for proposed sampling schemes, we also conduct the same experiment on rotated versions of the test signal $f_{\mathrm{t}}$. The rotation is applied by choosing Euler angles $(\alpha, \beta, \gamma)$ from uniform distributions, where $\alpha, \gamma \in[0,2 \pi)$ and $\beta \in[0, \pi]$, and then applying to the test $f_{\mathrm{t}}$ using the $z y z$ convention [8].

We also conduct the same experiments for the spatial antipodal sampling scheme. We plot the average value of $E_{\text {mean }}$ for the proposed scheme and spatial antipodal sampling scheme for different rotations of the test signal in Fig. 2. It is evident that the reconstruction error is smaller for proposed scheme than the spatial antipodal sampling scheme for all $1 \leq L \leq 25$. It can also be observed that the reconstruction error is practically rotationally invariant for both schemes, as the order of magnitude of $E_{\text {mean }}$ does not change depending the angle of rotation.

\section{B. Computational and Storage Efficiency}

The asymptotic computational complexity to compute the SHT for both the proposed and spatial antipodal sampling schemes is same, that is, $O\left(L^{4}\right)$, which can reduced to $O\left(L^{3}\right)$ if we pre-compute the $\mathbf{P}_{L}^{m}$ matrices [12], [13]. However, the proposed scheme is, in practise, more efficient as it does not compute the odd degree spherical harmonic coefficients, resulting in the FFT being computed $(L+1) / 2$ rather than $L$ times. Furthermore, the matrix $\mathbf{P}_{L}^{m}$ has a smaller dimensionality $\left(\mathbf{P}_{L}^{m}\right.$ is largest for $m=0$ with $\mathbf{P}_{L}^{0}$ of size $(L+1) / 2$ rather than $L$ ), which enables fast computation of matrix inversion involved in solving the system of linear equations given in (11).

We calculated the computation time in seconds, denoted by $\tau$, to carry out the SHT for the proposed and spatial antipodal sampling schemes. The time taken to compute the SHT of the complex band-limited test signal $f_{\mathrm{t}}$ is recorded and averaged over 1000 test signals. The experiment is performed using MATLAB running on a machine equipped with $3.4 \mathrm{GHz}$ Intel Core $i 7$ processor and $8 \mathrm{~GB}$ of RAM. We found that

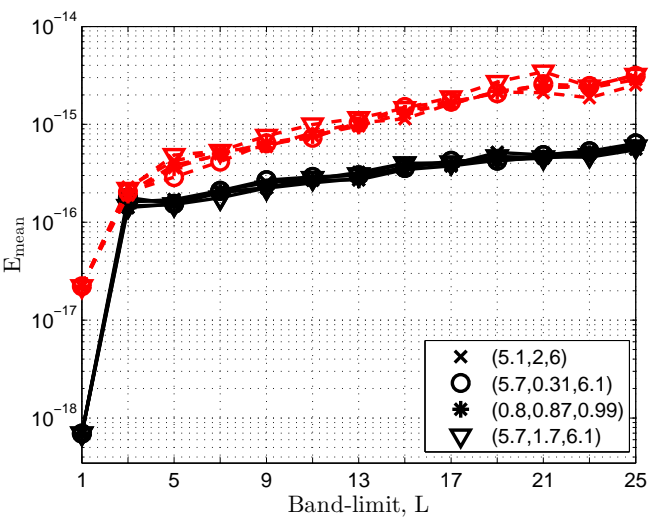

Fig. 2: Mean reconstruction error $E_{\text {mean }}$, given in (13), plotted in solid (black) and dashed (red) lines for the proposed sampling scheme and scheme in [13], respectively, for the original (without marker) test signal $f_{\mathrm{t}}$ and 4 rotated versions (indicated by markers) of the test signal and for bandlimits $1 \leq L \leq 25$. The rotation angles in radians are in the form of Euler angles $(\alpha, \beta, \gamma)$, as displayed in the legend, where the rotation is applied using the $z y z$ rotation convention [8].

the SHT associated with proposed scheme requires slightly less computation time, showing that while it has the same asymptotic computational complexity, it is more efficient in practise. The SHT has to be carried out for every voxel in an image; for an average sized male brain of $1260 \mathrm{~cm}^{3}$ with imaging done at a spatial resolution of $1.25 \mathrm{~mm}$ with $L=11$ ( $N_{\mathrm{O}}=66$, a typical number of samples for dMRI) [21], [22] the cumulative time for calculating the SHT once for every voxel is 1520 seconds for the proposed scheme and 1660 seconds for the spatial antipodal scheme. For large databases of subjects, such as the Human Connectome Project composed of 1200 subjects [22], the difference in computation time is significant.

Furthermore, the proposed scheme requires only $L(L+1) / 2$ rather than $L^{2}$ spherical harmonics to be computed, therefore reduces the storage space required to store the spherical harmonic representation of the diffusion signal by approximately half.

\section{CONCLusions}

We have developed a sampling scheme on the sphere for the reconstruction of the diffusion signal in dMRI that exploits the antipodal symmetry property of the diffusion signal in the spectral domain to attain an optimal number of samples. A sampling scheme that exploits the antipodal symmetry property of the diffusion signal in the spatial domain is the only other scheme that allows accurate reconstruction of the diffusion signal with the optimal number of samples. We have shown that the proposed sampling performs better than the spatial antipodal sampling scheme for the application of diffusion signal reconstruction in dMRI; the proposed sampling scheme will allow for more accurate diffusion signal reconstruction, as well as decreased processing time and storage requirements. 


\section{REFERENCES}

[1] E. Caruyer, C. Lenglet, G. Sapiro, and R. Deriche, "Design of multishell sampling schemes with uniform coverage in diffusion MRI," Magn. Reson. Med., vol. 69, no. 6, pp. 1534-1540, Jun. 2013.

[2] J. Cheng, D. Shen, and P.-T. Yap, "Designing single- and multipleshell sampling schemes for diffusion MRI using spherical code," in Med. Image Comput. Comput. Assist. Interv., MICCAI'2014, Boston, Massachusetts, 2014, vol. 8675, pp. 281-288.

[3] A. Daducci, J. D. McEwen, D. V. D. Ville, J. P. Thiran, and Y. Wiaux, "Harmonic analysis of spherical sampling in diffusion MRI," in Proc. 19th Ann. Meet. Int. Soc. Magn. Reson. Med., Jun. 2011.

[4] C. P. Hess, P. Mukherjee, E. T. Han, D. Xu, and D. B. Vigneron, "Qball reconstruction of multimodal fiber orientations using the spherical harmonic basis," Magn. Reson. Med., vol. 56, no. 1, pp. 104-117, Jul. 2006.

[5] D. K. Jones, M. A. Horsfield, and A. Simmons, "Optimal strategies for measuring diffusion in anisotropic systems by magnetic resonance imaging," Magn. Reson. Med., vol. 42, no. 3, pp. 515-525, Sep. 1999.

[6] C. G. Koay, "A simple scheme for generating nearly uniform distribution of antipodally symmetric points on the unit sphere," J. Comput. Sci., vol. 2, no. 4, pp. 377-381, Dec. 2011.

[7] W. Ye, S. Portnoy, A. Entezari, S. J. Blackband, and B. C. Vemuri, "An efficient interlaced multi-shell sampling scheme for reconstruction of diffusion propagators," IEEE Trans. Med. Imag., vol. 31, no. 5, pp. 1043-1050, May 2012.

[8] R. A. Kennedy and P. Sadeghi, Hilbert Space Methods in Signal Processing. Cambridge, UK: Cambridge University Press, Mar. 2013.

[9] D. K. Jones, T. R. Knösche, and R. Turner, "White matter integrity, fiber count, and other fallacies: The do's and don'ts of diffusion MRI," Neurolmage, vol. 73, pp. 239-254, Jun. 2013.

[10] E. Caruyer and D. Rachid, "A Computational Framework for Experimental Design in Diffusion MRI," in MICCAI Workshop on Computational Diffusion MRI, CDMRI'2012, Nice, France, Oct. 2012.

[11] H. E. Assemlal, D. Tschumperlé, L. Brun, and K. Siddiqi, "Recent advances in diffusion MRI modeling: Angular and radial reconstruction," Medical Image Analysis, vol. 15, no. 4, pp. 369-396, Aug. 2011.

[12] Z. Khalid, R. A. Kennedy, and J. D. McEwen, "An optimaldimensionality sampling scheme on the sphere with fast spherical harmonic transforms," IEEE Trans. Signal Process., vol. 62, no. 17, pp. 4597-4610, Sep. 2014.

[13] A. P. Bates, Z. Khalid, and R. A. Kennedy, "An optimal dimensionality sampling scheme on the sphere for antipodal signals in diffusion magnetic resonance imaging," in Proc. IEEE Int. Conf. Acoust., Speech, Signal Process., ICASSP'2015, Brisbane, Australia, 2015.

[14] K. Ugurbil, J. Xu, E. J. Auerbach, S. Moeller, A. T. Vu, J. DuarteCarvajalino, C. Lenglet, X. Wu, S. Schmitter, d. M. van, J. Strupp, G. Sapiro, F. De Martino, D. Wang, N. Harel, M. Garwood, L. Chen, D. A. Feinberg, S. M. Smith, K. L. Miller, S. N. Sotiropoulos, S. Jbabdi, J. L. R. Andersson, T. E. J. Behrens, M. F. Glasser, C. van Essen, David, and E. Yacoub, "Pushing spatial and temporal resolution for functional and diffusion mri in the human connectome project," NeuroImage, vol. 80, pp. 80-104, Oct 2013.

[15] M. Descoteaux, E. Angelino, S. Fitzgibbons, and R. Deriche, "Regularized, fast, and robust analytical q-ball imaging," Magn. Reson. Med., vol. 58, no. 3, pp. 497-510, Sep. 2007.

[16] D. S. Tuch, "Q-ball imaging," Magn. Reson. Med., vol. 52, no. 6, pp. 1358-1372, 2004.

[17] E. Caruyer, "Q-space diffusion MRI: acquisition and signal processing," thesis, Université Nice Sophia Antipolis, Jul. 2012.

[18] J. D. McEwen and Y. Wiaux, "A novel sampling theorem on the sphere," IEEE Trans. Signal Process., vol. 59, no. 12, pp. 5876-5887, Dec. 2011.

[19] J.-D. Tournier, F. Calamante, and A. Connelly, "Determination of the appropriate $\mathrm{b}$ value and number of gradient directions for high-angularresolution diffusion-weighted imaging," NMR Biomed., vol. 26, no. 12, pp. 1775-1786, Dec. 2013.

[20] E. Caruyer and R. Deriche, "Diffusion MRI signal reconstruction with continuity constraint and optimal regularization," Med. Image Anal., vol. 16, no. 6, pp. 1113-1120, Aug. 2012.

[21] A. Daducci, E. J. Canales-Rodrìguez, M. Descoteaux, E. Garyfallidis, Y. Gur, Y.-C. Lin, M. Mani, S. Merlet, M. Paquette, A. RamirezManzanares, M. Reisert, P. Reis Rodrigues, F. Sepehrband, E. Caruyer, J. Choupan, R. Deriche, M. Jacob, G. Menegaz, V. Prčkovska, M. Rivera, Y. Wiaux, and J. P. Thiran, "Quantitative comparison of reconstruction methods for intra-voxel fiber recovery from diffusion MRI," IEEE Trans. Med. Imag., vol. 33, no. 2, pp. 384-399, Feb. 2014.
[22] S. N. Sotiropoulos, S. Jbabdi, J. Xu, J. L. Andersson, S. Moeller, E. J. Auerbach, M. F. Glasser, M. Hernandez, G. Sapiro, M. Jenkinson, D. A. Feinberg, E. Yacoub, C. Lenglet, D. C. van Essen, K. Ugurbil, and T. E. Behrens, "Advances in diffusion MRI acquisition and processing in the Human Connectome Project," NeuroImage, vol. 80, pp. 125-143, Oct. 2013. 